\title{
AIDS: Signs to Management of the Disease
}

\author{
Singhal $\mathrm{C}^{1}$, Chauhan $\mathrm{N}^{1}$, Malhotra $\mathrm{N}^{2}$, Sunny ${ }^{1}$ and Narang $\mathrm{J}^{* 1}$ \\ ${ }^{1}$ Amity Institute of Nanotechnology, Amity University, Noida, India \\ ${ }^{2}$ Amity Institute of Physiotherapy, Amity University, Noida, India \\ *Corresponding author: Narang J, Amity Institute of Nanotechnology, Amity University, Noida, India, Tel: \\ +919811792572, E-mail: jags_biotech@yahoo.co.in
}

Citation: Singhal C, Chauhan N, Malhotra N, Sunny, Narang J (2015) AIDS: Signs to Management of the

Disease. J Aids Hiv Infec 1(1): 105. doi: 10.15744/2454-499X.1.105

Received Date: February 24, 2015 Accepted Date: April 07, 2015 Published Date: April 09, 2015

\begin{abstract}
Acquired Immune Deficiency Syndrome (AIDS) is a spectrum of conditions caused by infection with the human immunodeficiency virus (HIV). They get it after being infected with the HIV virus. HIV (human immunodeficiency virus) is a retrovirus that primarily infects components of the immune system. Symptoms of HIV are often mistaken for another viral infection, such as flu. Various diagnostic methods are available for detection of HIV. Among them, anti-HIV antibodies are the core of testing and diagnosis of HIV. Antiretroviral therapy (ART) is the recommended treatment for HIV infection. ART involves taking a combination (regimen) of three or more anti-HIV medications daily, thus, preventing HIV from multiplying and destroying infection-fighting CD4 cells and changing the course of the HIV epidemic.
\end{abstract}

Keywords: HIV; Retrovirus; Treatment; Diagnosis

\section{Introduction}

\section{Acquired Immune Deficiency Syndrome (AIDS)}

AIDS is the most advanced stages of HIV infection in which there is severe loss of body's cellular immunity, greatly lowering the resistance to infection or malignancy. It is caused by human immunodeficiency virus (HIV). In AIDS, Acquired means that people are not born with the disease. They get it after being infected with the HIV virus. Immuno means the immune system. The immune system is the part of the body that fights off disease. Deficiency refers to not enough. An immuno-deficiency is a problem where the immune system is damaged and cannot fight off diseases to keep the body healthy. Syndrome is a set of signs and symptoms that appear together and characterize a disease or medical condition [1-3]. HIV is a retrovirus. Retroviruses have RNA as their genetic material possessing an enzyme, called reverse transcriptase that gives them the unique property of transcribing their RNA into DNA after entering a cell. The retroviral DNA can then integrate into the chromosomal DNA of the host cell, to be expressed there. The virus primarily infects the components of the human immune system such as CD4+ $4^{+}$cells, macrophages and dendritic cells and directly or indirectly destroys majority of these cells. As a consequence, the human body can't fight off infections and disease which leads to AIDS [4,5]. The HIV-1 virion is an enveloped structure containing 72 external spikes. These spikes are formed by the two major viral-envelope proteins, gp120 and gp41. (Gp stands for glycoprotein-the proteins are linked to sugars-and the number refers to the mass of the protein, in thousands of daltons.) The HIV-1 lipid bilayer is also studded with various host proteins, including class I and class II major histocompatibility complex molecules, acquired during virion budding. The cone-shaped core of HIV-1 contains p17 as the matrix protein and p25, p6, p7 (or p9) as the nucleocapsid proteins each of which is proteolytically cleaved from a $53 \mathrm{kDa}$ gag precursor by the HIV-1 protease. The phosphorylated p25 polypeptide forms the chief component of the inner shelf of the nucleocapsid, whereas the p17 protein is associated with the inner surface of the lipid bilayer and stabilizes the exterior and interior components of the virion. The p7 protein binds directly to the genomic RNA through a zinc-finger structural motif and together with $\mathrm{p} 9$ forms the nucleoid core. The retroviral core contains two copies of the single-stranded HIV-1 genomic RNA that is associated with the various preformed viral enzymes, including the reverse transcriptase, integrase, ribonuclease, and protease [6].

\section{Signs of AIDS disease}

The stages of HIV infection are acute infection/primary stage, latency/asymptomatic stage and AIDS/symptomatic stage.

Primary HIV Infection: Primary infection with HIV occurs two to six weeks after infection. Various clinical studies show that many infected people experience flu-like symptoms during primary infection, which are ignored partly because of the selflimiting and mild nature of those symptoms. Clinical symptoms of primary infection, when recognized, may include mild fever, muscle aches and pains, fatigue, headaches, lymphadenopathy, rashes, a sore throat, mild diarrhea, myalgia, pharyngitis and less commonly, nausea, vomiting and neurological symptoms [7]. 
Clinical latency period/Asymptomatic Infections: This stage is marked by disappearance of symptoms of acute viral disease, down regulation of viraemia, CD4 cell count becomes almost normal and the neutralizing and complement (C1) fixing virus specific antibodies appear in the blood. Humoral immunity is intact during the asymptomatic stage that is specific antibodies are produced against different viral proteins, but the antibodies are not protective, are not able to interfere with cell to cell transmission and infectivity of virus on account of constant variation of virus. This period on average lasts for 8-10 years. Progressive impairment of HIV specific and nonspecific cell mediated and humoral immune responses heralds the onset of AIDS [8].

AIDS: Advanced stage of HIV infection is characterized by increase in all virological parameters (virus load, p24 antigen etc.) in both peripheral blood and lymph nodes. Lymphoid tissue is totally destroyed and replaced by fibrous tissue. Virus trapping by whatever lymphoid tissue remains is minimal or Nil. There is profound immune suppression and opportunistic infections may prove fatal at this stage. The CD4 count is usually less than 200 cells/ $\mu \mathrm{l}$ and progressively falls. People with AIDS also have an increased risk of developing various cancers such as Kaposi's sarcoma, cervical cancer and cancers of the immune system known as lymphomas. Additionally, people with AIDS often have systemic symptoms of infection like fevers, sweats (particularly at night), swollen glands, chills, weakness, and weight loss $[8,9]$.

\section{Transmission of HIV infection}

Risk factors for HIV infection includes multiple homosexual or heterosexual partners; contaminated blood transfusion; injections with contaminated needles and syringes and infected mother to foetus/infant (before, during/shortly after birth). The efficiency of transmission of HIV is determined by the amount of virus in the body fluid and the extent of contact. High concentrations of free infectious virus and virus infected cells have been reported in blood, genital fluids and cerebrospinal fluids. Breast milk and saliva yield varying numbers, whereas, other body fluids have a low viral content. Saliva in adults contains some non-inhibitory substances like fibronectins and glycoproteins which could prevent cell to cell transfer of virus. Thus, saliva is not likely a vehicle of transmission. Urine, sweat, milk, broncho-alveolar lavage fluid, amniotic fluid, synovial fluid, faeces and tears have been reported to yield zero or a few HIV particles. Hence, these vehicles also do not appear to be important in vehicle transmission. Breast milk at the time of primary infection in a feeding mother has a high content of virus and may transmit the infection to the baby. Cerebrospinal fluid (CSF), on the other hand, also has a high content of virus particularly in individuals with neurological disease, but, CSF is not a natural source of virus transmission [9] Scheme1.

\section{HIV virus (Human Being is the reservoir or host)}

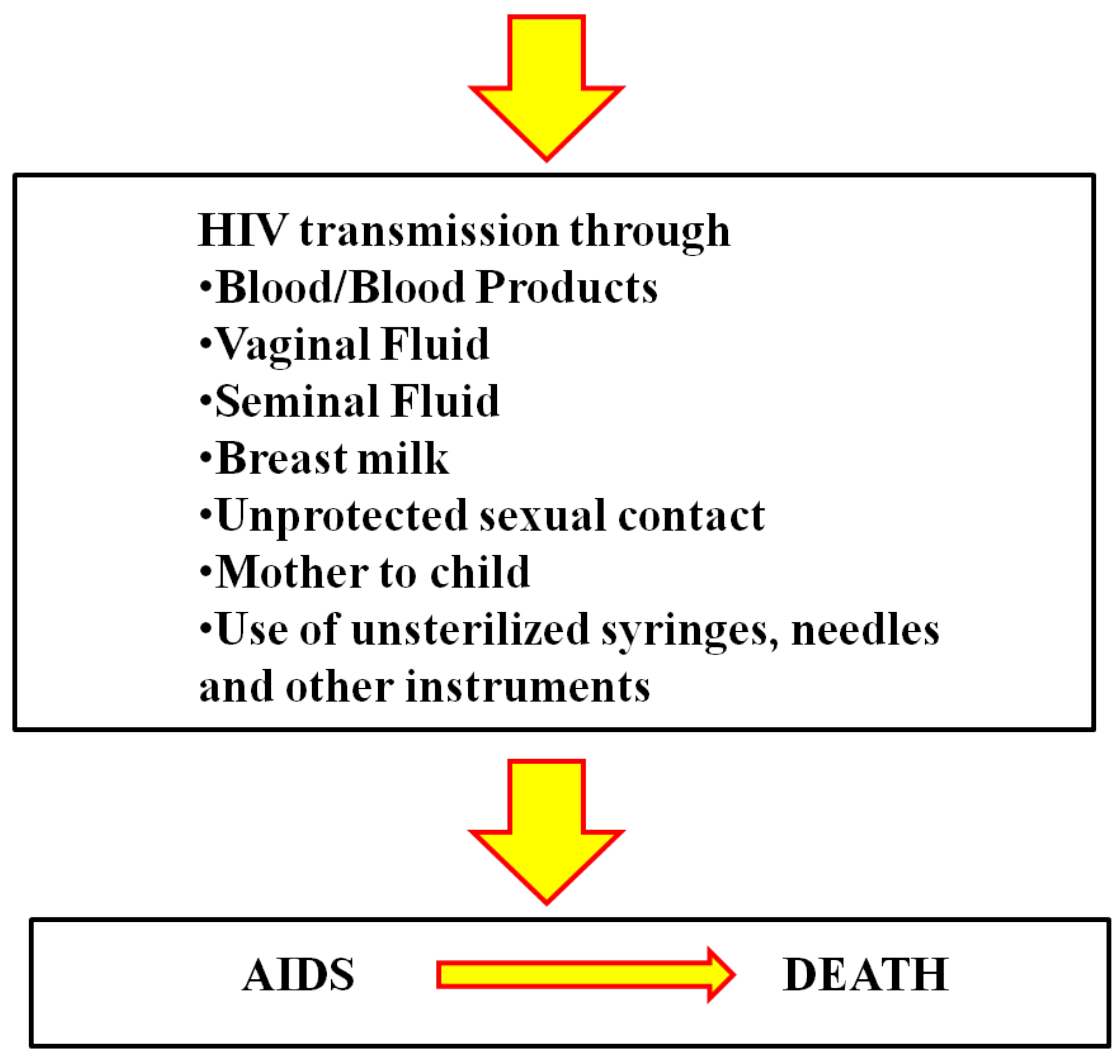

Scheme1: Schematic representation showing transmission of disease

Analysis of AIDS and HIV infection with the help of various tests 
It is clear that $20-80 \%$ people in the world who have HIV do not know their HIV status. It is therefore important to make use of every opportunity to offer to test people who are unaware of their status [10]. The testing laboratories play a major role in testing HIV with the help of proper diagnostic protocols [9]. The testing and diagnosis of HIV can be done with the help of detection of anti-HIV antibodies.

The tests which help to detect specific HIV antibodies can be classified into:

Screening Test: The screening tests can be can broadly be broadly classified into:

1. ELISA: Indirect ELISA, Competitive ELISA, Sandwich ELISA, Capture Assays.

Though ELISA is easy to perform, highly sensitive, specific and cost effective; but there a certain drawbacks in performing ELISA. One is that it is time consuming (takes 2-3 hours) and another is that it follows a confirmatory test.

2. Agglutination Assays

3. Dot blot assays/Coomb test

4. Immunochromatography

Apart from ELISA all of the above screening tests are RAPID but the cost of their performance is very high.

Supplemental/Confirmatory Tests:

1. Western Blot (WB)/ Immunoblot/ Line Immunoassay: It is highly specific but expensive.

2. Indirect Fluorescent antibody test (IFA)

3. Radioimmunoprecipitation test (RIPA)

IFA and RIPA are highly specific but these techniques are not used in India because of their very high cost.

4. Nucleic Acid Amplification Test (NAAT): It helps in early infant diagnosis.

5. HIV p24 antigen test: Helps to diagnose early HIV infection [11].

\section{Management of AIDS}

The treatment of human immunodeficiency virus (HIV) disease depends on the stage of the disease and any concomitant opportunistic infections [12]. In general, the goal of treatment is to prevent the immune system from deteriorating to the point that opportunistic infections become more likely. Immune reconstitution syndrome is also less likely in patients whose immune systems are weakened to this point. In 1987, a drug called Azidothymidine (AZT) became the first approved treatment for HIV disease. Since then, approximately 30 drugs have been approved to treat people living with HIV/AIDS, and more are under development.

There are currently five different "classes" of HIV drugs. Each class of drug attacks the virus at different points in its life cycle. Generally cocktails of these antiretrovirals are taken:

Nucleoside/ Nucleotide reverse transcriptase inhibitors (NRTIs): Sometimes called "nukes". These drugs work to block a very important step in HIV's reproduction process. Nukes act as faulty building blocks in production of viral DNA production. This blocks HIV's ability to use a special type of enzyme (reverse transcriptase) to correctly build new genetic material (DNA) that the virus needs to make copies of itself [13].

Non-nucleoside reverse transcriptase inhibitors (NNRTIs): These are called "non-nukes." They work in a very similar way to "nukes." Non-nukes also block the enzyme, reverse transcriptase, and prevent HIV from making copies of its own DNA. EPT [14] and TIBO [15] derivatives were the first two classes of compounds that could be categorized as NNRTIs, i.e. non-nucleoside HIV-1 RT inhibitors.

Protease inhibitors (PIs): When HIV replicates inside your cells, it creates long strands of its own genetic material. These long strands have to be cut into shorter strands in order for HIV to create more copies of themselves. The enzyme that acts to cut up these long strands is called protease. Protease inhibitors (stoppers) block this enzyme and prevent those long strands of genetic material from being cut up into functional pieces. The ten PIs presently available for the treatment of HIV infections are saquinavir, ritonavir, indinavir, nelfinavir, amprenavir, lopinavir, atazanavir, fosamprenavir, tipranavir and darunavir. Darunavir was the tenth and, so far, last PI to reach the market [16-18].

Entry/Fusion inhibitors (FIs): These medications work to block the virus from ever entering your cells in the first place. HIV needs a way to attach and bond to your CD4 cells, and it does that through special structures on cells called receptorsites. Receptor sites are found on both HIV and CD4 cells (they are found on other types of cells too). Fusion inhibitors can target those sites on either HIV or CD4 cells and prevent HIV from "docking" into your healthy cells. There is one fusion inhibitor (FI) currently available for the treatment of HIV infections, i.e., enfuvirtide [13].

Integrase inhibitors (INIs): HIV uses your cells' genetic material to make its own DNA (a process called reverse transcription). Once that happens, the virus has to integrate its genetic material into the genetic material of your cells. This is accomplished by an enzyme called integrase. Integrase inhibitors block this enzyme and prevent the virus from adding its DNA into the DNA in your CD4 cells. Preventing this process prevents the virus from replicating and making new viruses. The first integrase inhibitor (INI) licensed for clinical use, raltegravir, has only recently (in 2007) been approved and it has proven highly effective in reducing viral loads in HIV-infected patients [19-21]. 
Anti-HIV drug combinations: highly active antiretroviral therapy (HAART): Highly active antiretroviral therapy (HAART) is the principal method for preventing immune deterioration. In addition, prophylaxis for specific opportunistic infections is indicated in particular cases.

Successful long-term HAART results in a gradual recovery of CD4 T-cell numbers and an improvement of immune responses and T-cell repertoire (previously lost antigen responses may be restored). The peripheral T-cell counts initially surge after therapy is initiated, but this represents redistribution of activated $\mathrm{T}$ cells from the viral replication centers in the lymph nodes rather than a true increase in total-body CD4 T-cell counts [22,23].

In addition to virologic response and reduced risk of opportunistic infection, there is evidence to suggest that non-AIDS-defining illnesses, in particular psychiatric and renal disease, may also be reduced when on HAART. Although multifactorial in nature (transmission mode and patient educational level are independent risk factors for these events) there may also be a direct role of HIV in these events, or an indirect role mediated through the subsequent immune dysfunction. Some non-AIDS-defining illnesses, such as liver and cardiovascular disease, are not improved by HAART [24].

\section{Conclusion}

Acquired immune deficiency syndrome (AIDS) isn't a disease in itself; rather, AIDS is a condition that develops when a person's body has been weakened by HIV (human immunodeficiency virus). Following initial infection, a person may experience a brief period of influenza-like illness. This is typically followed by a prolonged period without symptoms. Many governments and research institutions participate in AIDS research. This research includes behavioral health interventions such as sex education, and drug development, such as research into microbicides for sexually transmitted diseases, HIV vaccines, and antiretroviral drugs. Other medical research areas include the topics of pre-exposure prophylaxis, post-exposure prophylaxis, and circumcision and HIV.

\section{References}

1. Sepkowitz KA (2001) AIDS-the first 20 years. N Engl J Med 344: 1764-72.

2. Krämer A, Kretzschmar M, Krickeberg K (2010) Modern Infectious Disease Epidemiology Concepts, Methods, Mathematical Models and Public Health. Springer 88 .

3. Kirch W (2008) Encyclopedia of public Health. Springer 676-7.

4. Centers for Disease Control and Prevention/HIV/Basics.

5. Alimonti JB, Ball TB, Fowke KR (2003) Mechanisms of CD4+ T lymphocyte cell death in human immunodeficiency virus infection and AIDS. J Gen Virol 84: 1649-61.

6. Prescott, Harley, Klein (2002) Microbiology (5 $5^{\text {th }}$ Edition) The McGraw-hill companies.

7. Fauci AS (1996) Host factors and pathogenesis of HIV 1 induced disease. Nature 384: 529-34.

8. Guss DA (1994) The acquired immune deficiency syndrome: an overview for the emergency physician Part 2. J Emerg Med 12: $491-97$.

9. Guidelines on HIV testing (2007) National AIDS Control Organisation Ministry of Health and Family Welfare, NACO.

10. Marks G, Crepaz N, Senterfitt JW, Janssen RS (2005) Meta-Analysis of high-risk sexual behavior in persons aware and unaware they are infected with HIV in the United States: implications for HIV prevention programs. J Acquir Immune Defic Syndr 39: 446-53.

11. Ridderhof JC, Deun VA, Kam KM, Narayanan PR, Aziz MA (2007) Roles of laboratories and laboratory systems in effective tuberculosis programmes. Bull World Health Organ 85: 354-9.

12. Panel on Antiretroviral Guidelines for Adults and Adolescents. Guidelines for the use of antiretroviral agents in HIV-1-infected adults and adolescents.

13. Clercq E (2009) Anti-HIV drugs: 25 compounds approved within 25 years after the discovery of HIV. Int J Antimicrob Agents 33 : $307-20$.

14. Baba M, Tanaka H, De Clercq E, Pauwels R, Balzarini J, et al. (1989) Highly specific inhibition of human immunodeficiency virus type 1 by a novel 6-substituted acyclouridine derivative. Biochem Biophys Res Commun165: 1375-81.

15. Pauwels R, Andries K, Desmyter J, Schols D, Kukla MJ, et al. (1990) Potent and selective inhibition of HIV-1 replication in vitro by a novel series of TIBO derivatives. Nature 343: 470-4.

16. Pauwels R (2006) Aspects of successful drug discovery and development. Antiviral Res 71: 77-89.

17. Madruga JV, Cahn P, Grinsztejn B, Haubrich R, Lalezari J, et al. (2007) Efficacy and safety of TMC125 (etravirine) in treatment-experienced HIV-1-infected patients inDUET-1: 24-week results froma randomised, double-blind, placebocontrolled trial. Lancet 370: 29-38.

18. Lazzarin A, Campbell T, Clotet B, Johnson M, Katlama C, et al. (2007) Efficacy and safety of TMC125 (etravirine) in treatment-experienced HIV-1-infected patients inDUET-2: 24-week results from a randomised, double-blind, placebo-controlled trial. Lancet 370: 39-48.

19. Grinsztejn B, Nguyen BY, Katlama C, Gatell JM, Lazzarin A, et al. (2007) Safety and efficacy of the HIV-1 integrase inhibitor raltegravir (MK-0518) in treatmentexperienced patients with multidrug-resistant virus: a phase II randomized controlled trial. Lancet 369: 1261-9.

20. Steigbigel RT, Cooper DA, Kumar PN, Eron JE, Schechter M, et al. (2008) Raltegravir with optimized background therapy for resistant HIV-1 infection. N Engl J Med 359: 339-54.

21. Cooper DA, Steigbigel RT, Gatell JM, Rockstroh JK, Katlama C, et al. (2008) Subgroup and resistance analyses of raltegravir for resistant HIV-1 infection. N Engl J Med 359: 355-65.

22. Bucy RP, Hockett RD, Derdeyn CA, Saag MS, Squires K et al. (1999) Initial increase in blood CD4(+) lymphocytes after HIV antiretroviral therapy reflects redistribution from lymphoid tissues. J Clin Invest 103:1391-8. 
23. Pakker NG, Notermans DW, de Boer RJ, Roos MT, de Wolf F, et al. (1998) Biphasic kinetics of peripheral blood T cells after triple combination therapy in HIV-1 infection: a composite of redistribution and proliferation. Nat Med 4: 208-14.

24. Masia M, Padilla S, Alvarez D, López JC, Santos I, et al. (2013) Risk, predictors, and mortality associated with non-AIDS events in newly diagnosed HIV-infected patients: role of antiretroviral therapy. AIDS 27: 181-9.

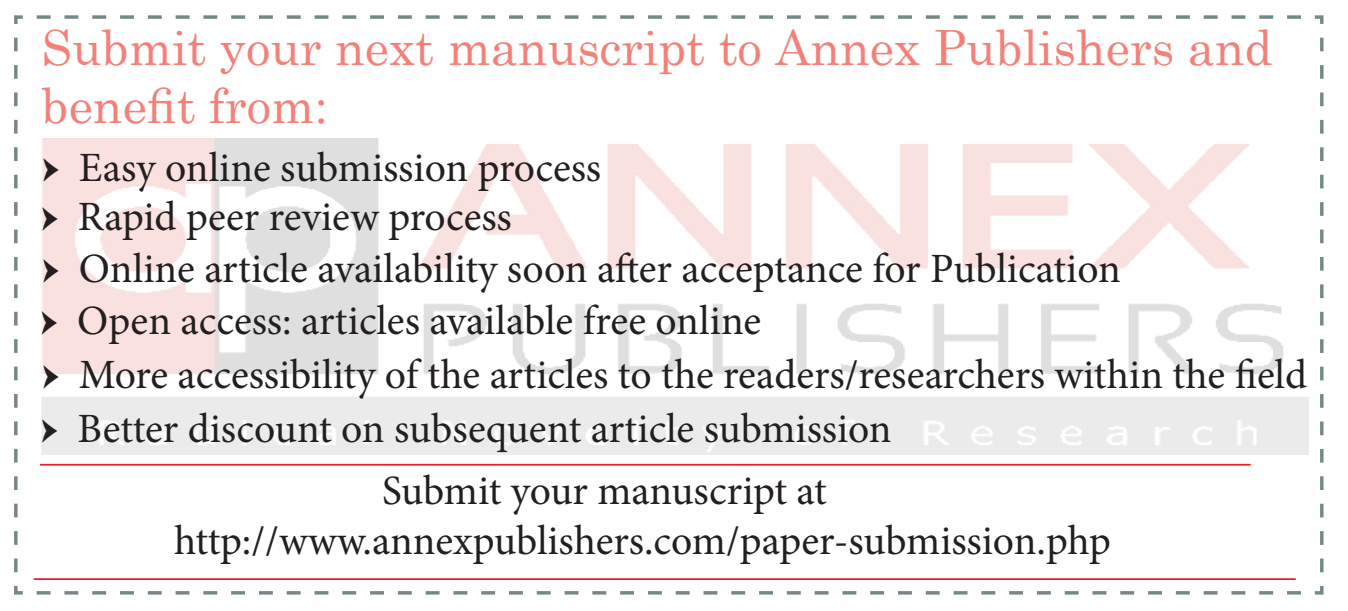

